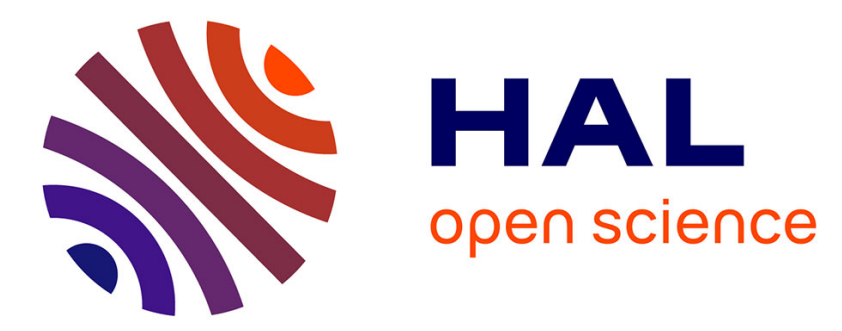

\title{
ROI-BASED RATE CONTROL USING TILES FOR AN HEVC ENCODED VIDEO STREAM OVER A LOSSY NETWORK
}

\author{
Marwa Meddeb, Marco Cagnazzo, Béatrice Pesquet-Popescu
}

\section{To cite this version:}

Marwa Meddeb, Marco Cagnazzo, Béatrice Pesquet-Popescu. ROI-BASED RATE CONTROL USING TILES FOR AN HEVC ENCODED VIDEO STREAM OVER A LOSSY NETWORK. Image Processing (ICIP), 2015 IEEE International Conference on, Sep 2015, Quebec city, Canada. hal01187296

\author{
HAL Id: hal-01187296 \\ https://hal.science/hal-01187296
}

Submitted on 27 Aug 2015

HAL is a multi-disciplinary open access archive for the deposit and dissemination of scientific research documents, whether they are published or not. The documents may come from teaching and research institutions in France or abroad, or from public or private research centers.
L'archive ouverte pluridisciplinaire HAL, est destinée au dépôt et à la diffusion de documents scientifiques de niveau recherche, publiés ou non, émanant des établissements d'enseignement et de recherche français ou étrangers, des laboratoires publics ou privés. 


\title{
ROI-BASED RATE CONTROL USING TILES FOR AN HEVC ENCODED VIDEO STREAM OVER A LOSSY NETWORK
}

\author{
Marwa Meddeb ${ }^{1}$, Marco Cagnazzo ${ }^{1}$, and Béatrice Pesquet-Popescu ${ }^{1}$ \\ ${ }^{1}$ Institut Mines-Télécom; Télécom ParisTech; CNRS LTCI
}

\begin{abstract}
The growth in the use of high definition (HD) and above video resolutions streams has outstripped the rate at which network infrastructure has been deployed. Video streaming applications require appropriate rate control techniques that make use of the specific characteristics of the video content, such as the regions of interest (ROI). With the introduction of high efficiency video coding (HEVC) streams, we consider new coding features to make a novel ROI-based rate control (RC) algorithm. The proposed approach introduces tiling in a ROI-based rate control scheme. It aims at enhancing the quality of important regions (i.e. faces for a videoconferencing system) considering independently coded regions lying within an ROI and helps evaluating the ROI quality under poor channel conditions. Our work consists of two major steps. First, we designed a RC algorithm based on an independent processing of tiles of different regions. Second, we investigate the effect of ROI- and tile-based rate control algorithm on the decoded quality of the stream transmitted over a lossy channel.
\end{abstract} loss

Index Terms - HEVC, Tiles, Rate control, ROI coding, Packet

\section{INTRODUCTION}

Rate control (RC) plays an important role in video coding technologies. It helps to deal with bit rate and compressed media quality fluctuations. RC methods have been widely studied and suitable schemes have been developed for specific applications [1] and different video coding standards such as TM5 for MPEG-2, TMN8 for H.263 and VM8 for MPEG-4.

Recently with rapid demands for region of interest (ROI) in applications such as videoconferencing systems, video surveillance and telemedicine, ROI-based RC has become an interesting feature to study. The subjective visual quality mainly depends on some important areas. Therefore, many contributions have introduced RC algorithms aiming at improving the quality in the ROIs. Different techniques based on region level bit allocation and independent ratequantization (R-Q) models have been introduced in H.264/AVC to compute a quantization parameter $(\mathrm{QP})$ per region [2-5].

Meanwhile, the new HEVC standard has been finalized by ITU-T and ISO/IEC [6] and many works have focused on RC and developed new R-Q schemes for it. All the above-mentioned ROI-based RC algorithms were specially designed for their corresponding coding standards, and may not be efficient for HEVC. Therefore, we propose in this paper a new ROI-based rate control scheme for videoconferencing systems taking into account new features introduced in HEVC standard such as tiling. This feature has been introduced in HEVC for parallel processing, but can be useful in ROI based applications [7]. Tiles are used in our work to have independently decodable regions and two different kind of streams (ROI and non-ROI streams).
This work is an improvement of previously proposed scheme for H.264/AVC [2-5] and of our ROI-based controller designed for HEVC [8] [9]. Referring to our previous work based on the $R-\lambda$ RC model [10] [11] introduced in HM.13 [12], we added a tilling structure for picture partitioning to our ROI-based controller. The reference RC algorithm is enhanced with three main features; first, using an object detection method, we detect our ROI and generate automatically a binary map (ROI map). The frame is divided into tiles of different sizes, each tile containing only the coding tree units (CTUs) of the same region. A second binary map is then generated assigning each tile to the corresponding region. The target bit rate is allocated among regions considering a fixed weight. Then, the QP of each CTU is computed referring to the rate model of the associated region, the allocated bit budget and the corresponding tile. Finally, the proposed method considers independent rate-distortion (R-D) models for independently decodable regions over tiles, taking into account the importance of the ROI.

After encoding the sequence, a transmission and decoding simulation is performed to demonstrate the effect of the proposed ROIand tile-based controller. The obtained bitstream is transmitted over a lossy network modelized by a Gilbert-Elliott channel which is a simple and reliable representation of lossy networks [13] [14]. At the receiver side, a simple error concealment algorithm is proposed to reconstruct the corrupted video stream.

Overall, we show that the quality of the ROI is improved and the bit rate limit is respected, when using ROI- and tile-based RC algorithm. Thanks to tiles, the errors are not propagated in the whole frame, they are only limited in the corresponding region and the impact of packet loss on ROI quality is significantly diminished.

The remainder of the paper is organized as follows. Section 2 briefly reviews the main HEVC features that we consider in our work: rate contol, tilling and bitstream transmission. Then, in Section 3, the proposed algorithms and the performed experiments are explained and detailed. In Section 4 some experimental results are presented. Finally, conclusions and future research directions are given in Section 5 .

\section{RELATED WORKS}

\subsection{Rate control in HEVC}

In the HEVC reference software (HM) two different RC algorithms have been proposed. The first one is based on a quadratic ratedistortion model and the mean absolute difference (MAD) between the original and the reconstructed signal [15] [16]. In the second algorithm, an $R-\lambda$ model that takes into account the hierarchical coding structure has been adopted [10]. This model, initially introduced in version 10 of the reference software (HM.10), has been improved in a more recent version (HM.13). Adaptive bit allocation at frame level has been introduced [11] by considering variable weights for each 
hierarchical level, that depend on video content characteristics. Then, in [17], the Intra frame rate control has been modified by enabling bit allocation and QP computing at CTU level. All these features have been used in our previous works to perform a ROI-based bit allocation [8] [9]. The scheme has been tested for different configurations which demonstrate the effectiveness of the controller. It achieves accurate bit rates and provides an improvement in the ROI quality. However, as it does not allow independently decodable regions and separate streams, the ROI is affected by the quality decrease of the non-ROI. This work is an improvement of the previously proposed techniques.

\subsection{Bitstream and picture formats and partitionings}

At video coding layer (VCL), HEVC introduces different partitioning structures: slices, slice segments and tiles. Both slices and tiles are used to enhance the parallel processing capability. Slices contain CTUs in raster scan order within a picture, while tiles are always rectangular regions of CTUs [18].

At network abstraction layer (NAL), a slice segment is defined as a sequence of CTUs ordered consecutively in the tile scan. A slice is partitioned in slice segments that fit the MTU size. Each slice segment is then encapsulated in a NAL unit [19]. The bitstream organization in terms of syntax and its encapsulation into the network layer units is based on slices. The relation between a slice segment and a tile can be deduced from the picture parameter set (PPS) of the NAL unit.

\subsection{Use of tiles in HEVC}

As said before a picture coded using HEVC may be partitioned into tiles which are rectangular sets of CTUs. They are independently decodable regions. This new feature introduced in HEVC standard offers a flexible classification of CTUs, a higher pixel correlation compared to slice [6] and a better coding efficiency as tiles do not contain header informations.

The benefits of tiling have been assessed in [7]. First, tiles offer better R-D performance in case of high level parallelization. Second, they facilitate improved maximum transmission unit (MTU) size matching comparing to traditional slices. Then, tiling can be used for additional ROI functionality, to ensure that the ROI tiles are independently decodable from the non-ROI tiles and that temporal and spatial predictions within the ROI do not refer to pixels outside the ROI [20]. ROI tile sections have been studied in different works to ensure a good fitting of the region and its corresponding tile [21]. They can be used for a tiled streaming for zoomable video, where all tiles are temporally aligned for an efficient bandwidth utilization and ROI quality improvement [22].

\section{PROPOSED APPROACH: TILING FOR ROI-BASED RATE CONTROL}

This section describes a new approach in ROI-based rate control initially introduced in [9] that takes into account tiling for region partitioning to ensure an improvement of the ROI encoding and transmission over the network.

\subsection{RC at Video coding layer}

At the VCL layer, we proposed a tile partition of the different sequences of class E ("Johnny", "KristenAndSara", "FourPeople") to identify ROIs as in the example represented in Fig.1.
The figure illustrates how each tile is classified as ROI or nonROI. Tiles are processed in raster scan order and, inside each tile, CTUs are also processed in raster scan order (see Fig.1b).

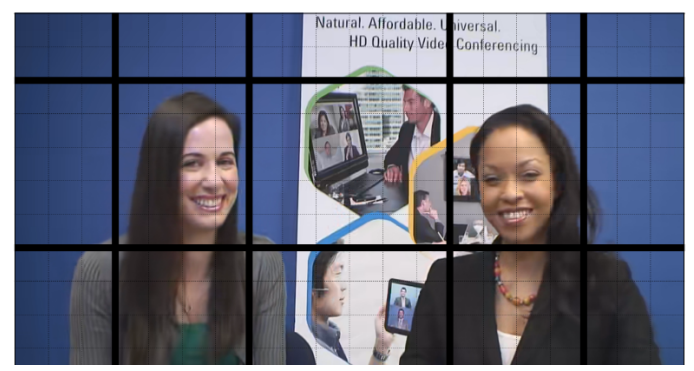

(a)

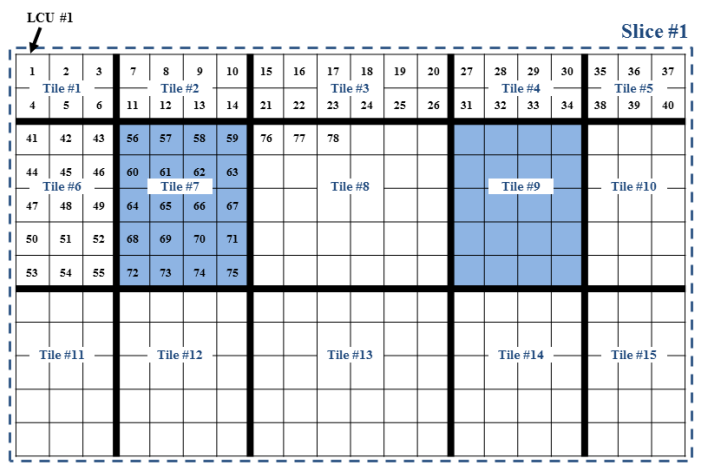

(b)

Fig. 1. Tile partitioning of "KristenAndSara": ROIs are in blue

The structure of the proposed HEVC controller differs significantly from the reference $R-\lambda$ algorithm [10], as we introduce a new level for region bit allocation and QP computing. At this level called "region level", the number of bits allocated per frame is allocated between the two regions, considering a factor $K$ as done in our previous work [9]. These bit budgets of the ROI and non-ROI are used independently to compute the number of encoding bits of CTUs of each region. Two independent $R-\lambda$ models are then used for ROI and non-ROI. Thus, the allocated bits per CTU is used as input to the R-D model of the corresponding region, to assign a QP per CTU.

The novelty in the current work consists in independent rate allocation and decoding of the ROI and the non-ROI, possible as they cover separate tiles. Thus, the ROIs are not affected by the quality decrease of the non-ROI obtained after the separated bit allocation performed between regions.

\subsection{Adaptation at Network abstraction layer}

Fig. 2 represents the number of allocated bits per tile (15 tiles in the given example), while encoding "KristenAndSara" sequence at low (a) and high (b) bit rates using the reference controller and tiling. Each line corresponds to an encoded frame (600 frames per sequence). The figure shows that the number of bits to encode different tiles (for both I and B frames) is not homogeneous, as tiles have different sizes in the proposed partitioning. Moreover, the number of bits per tile may exceed the MTU size of the network.

By matching tiles and slice segments it is possible to divide each tile into data streams to fit the MTU size (12000 bits for IP network and 800 bits for wireless environment) [23]. Thus, tiles of the ROI 


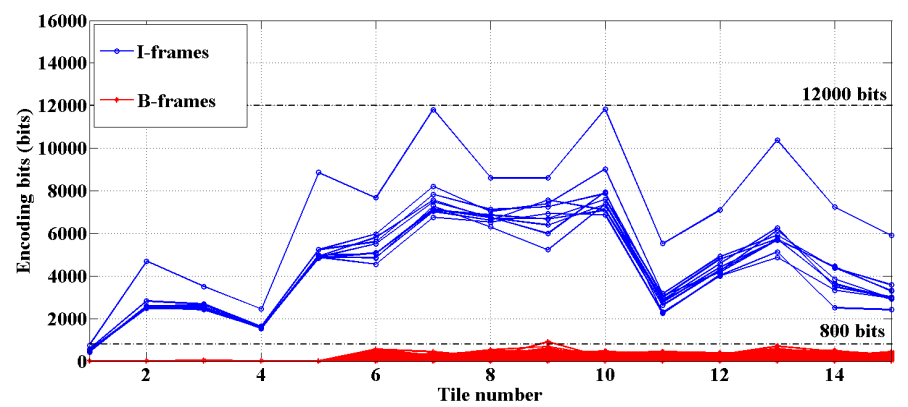

(a) $128 \mathrm{kbps}$

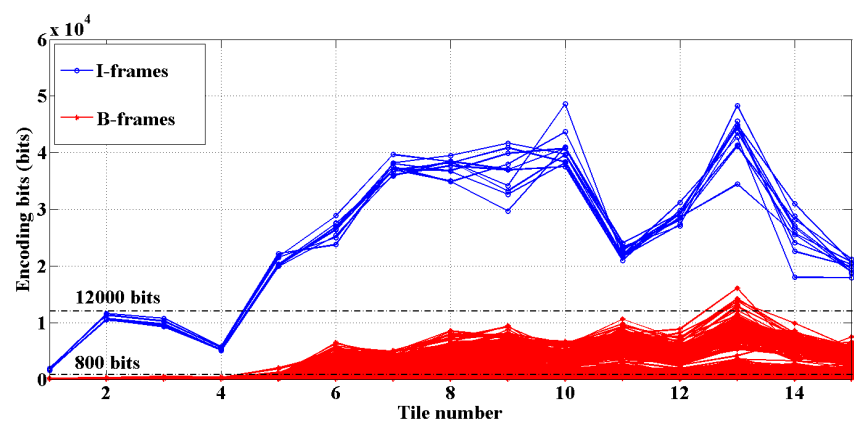

(b) $1.5 \mathrm{Mbps}$

Fig. 2. Number of encoding bits per tile ("KristenAndSara" sequence) at low and high bit rates

and tiles of the rest of the frame (non-ROI) would be encapsulated in different NAL units. The video stream would contain two kinds of NALs to transmit in the channel. If we consider the partitioning presented in Fig. 1 and if the number of bits allocated per tile does not exceed the MTU size, tile number 6 and tile number 7 will be encapsulated in separate NALs as illustrated in Fig.3.

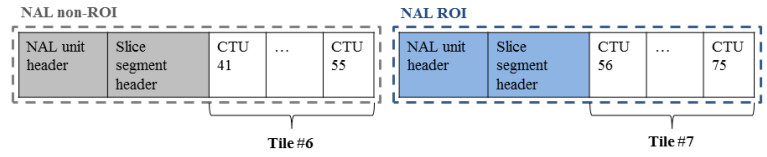

Fig. 3. NAL unit formats

\subsection{Packet loss and error concealment algorithm}

Each NAL unit is then packetized and transmitted over Gilbert-Elliott channels. For our experiments we use parameters taken from the literature [14]. Two algorithms are proposed and compared to show the effectiveness of ROI- and tile-based controller:

- No ROI nor tile based coding: If one packet is lost, all the following packets of the same frame are lost and all dependent frames are not decoded. Considering temporal dependencies between successive images, error is propagated till the next intra frame. At the decoder side, each corrupted frame is replaced with the last correctly decoded frame.

- ROI- and tile-based coding: Since packets of the same tile are dependent, we decide that if one packet of a tile is lost, all packets of the same tile are considered as lost. Considering temporal dependencies between tiles in the same spatial position, error is propagated inside tiles of the same position till the next intra frame. Consequently, at the decoder side, each corrupted or lost tile is replaced with the tile of the last decoded frame and at the same spatial position. This is a simple way to conceal errors in corrupted streams.

\section{EXPERIMENTAL RESULTS}

The proposed algorithm has been implemented in HM.13. We tested class E sequences ("Johnny", "KristenAndSara", "FourPeople") with the resolution of $1280 \times 720$ pixel, a frame rate equal to $60 \mathrm{fps}$ and 600 frames per sequence [24]. We used a low delay configuration as the algorithm is designed for videoconferencing systems. We used open GOPs of size 4 and an intra period equal to 60 to limit temporal error propagation. This section introduces two major results: R-D performance of the ROI- and tile-based controller and the quality of the decoded video stream partitioned into tiles.

\subsection{ROI- and tile-based controller performance}

We introduce the region bit allocation at two levels; at frame level to initialize a target amount of bits for each region, and at CTU level to make independent bit allocation of CTUs of different regions. At frame level, a positive constant $K$ is selected. It represents the desired ratio between the ROI and non-ROI bit rates:

$$
R_{r}=K \times R_{n}
$$

where $R_{r}$ and $R_{n}$ denote respectively the number of bits per pixel of the ROI and the non-ROI [9].

Table 1 gives the R-D performances for the three tested sequences and at low (128 kbps) and high (1.5 Mbps) bit rates, at various $K$ factors, together with the improvement in the ROI quality. One can also remark that the budget limit is respected with good accuracy. Moreover, for repartitioning factors $K$ bigger than the reference (gray lines), the quality of the ROI is improved. At low bit rates, we can have an increase in ROI peak signal-to-noise ratio (PSNR) up to $1.5 \mathrm{~dB}$.

\begin{tabular}{|c|c|c|c|c|c|c|c|}
\hline \multicolumn{4}{|c|}{ "Johnny" sequence at $128 \mathrm{kbps}$} & \multicolumn{4}{|c|}{ "Johnny" sequence at $1.5 \mathrm{Mbps}$} \\
\hline $\mathrm{K}$ & $\begin{array}{l}\text { Bitrate } \\
\text { (kbps) }\end{array}$ & $\begin{array}{c}\text { PSNR } \\
(\mathrm{dB})\end{array}$ & $\begin{array}{c}\Delta \text { PSNR } \\
\text { ROI (dB) }\end{array}$ & $\mathrm{K}$ & $\begin{array}{l}\text { Bitrate } \\
\text { (kbps) }\end{array}$ & $\begin{array}{c}\text { PSNR } \\
\text { (dB) }\end{array}$ & $\begin{array}{c}\Delta \text { PSNR } \\
\text { ROI (dB) }\end{array}$ \\
\hline 0.85 & 128.01 & 35.35 & & 0.81 & 1500.03 & 42.78 & \\
\hline 1.11 & 128.02 & 35.59 & 0.87 & 1.67 & 1498.22 & 42.72 & 0.07 \\
\hline 1.33 & 127.98 & 35.66 & 1.37 & 2.02 & 1493.17 & 42.68 & 0.04 \\
\hline 1.41 & 127.95 & 35.68 & 1.52 & 2.25 & 1487.54 & 42.64 & 0.01 \\
\hline \multicolumn{4}{|c|}{ "KristenAndSara" sequence at $128 \mathrm{kbps}$} & \multicolumn{4}{|c|}{ "KristenAndSara" sequence at $1.5 \mathrm{Mbps}$} \\
\hline $\mathrm{K}$ & $\begin{array}{l}\text { Bitrate } \\
\text { (kbps) }\end{array}$ & $\begin{array}{c}\text { PSNR } \\
(\mathrm{dB})\end{array}$ & $\begin{array}{c}\Delta \text { PSNR } \\
\text { ROI }(\mathrm{dB})\end{array}$ & $\mathrm{K}$ & $\begin{array}{l}\text { Bitrate } \\
\text { (kbps) }\end{array}$ & $\begin{array}{c}\text { PSNR } \\
(\mathrm{dB})\end{array}$ & $\begin{array}{c}\Delta \text { PSNR } \\
\text { ROI }(\mathrm{dB})\end{array}$ \\
\hline 0.95 & 130.59 & 32.38 & & 0.92 & 1500.27 & 42.58 & \\
\hline 1.16 & 129.56 & 32.58 & 0.60 & 1.66 & 1499.67 & 42.50 & 0.17 \\
\hline 1.37 & 129.00 & 32.65 & 0.97 & 1.89 & 1498.42 & 42.46 & 0.19 \\
\hline 1.47 & 128.77 & 32.68 & 1.00 & 1.99 & 1496.83 & 42.43 & 0.21 \\
\hline \multicolumn{4}{|c|}{ "FourPeople" sequence at $128 \mathrm{kbps}$} & \multicolumn{4}{|c|}{ "FourPeople" sequence at $1.5 \mathrm{Mbps}$} \\
\hline $\mathrm{K}$ & $\begin{array}{l}\text { Bitrate } \\
(\mathrm{kbps})\end{array}$ & $\begin{array}{c}\text { PSNR } \\
(\mathrm{dB})\end{array}$ & $\begin{array}{c}\Delta \text { PSNR } \\
\text { ROI (dB) }\end{array}$ & $\mathrm{K}$ & $\begin{array}{l}\text { Bitrate } \\
\text { (kbps) }\end{array}$ & $\begin{array}{c}\text { PSNR } \\
(\mathrm{dB})\end{array}$ & $\begin{array}{c}\Delta \text { PSNR } \\
\text { ROI (dB) }\end{array}$ \\
\hline 2.05 & 136.09 & 28.85 & & 2.07 & 1499.96 & 41.31 & \\
\hline 2.40 & 138.38 & 29.02 & 0.43 & 2.78 & 1499.68 & 41.22 & 0.15 \\
\hline 2.83 & 130.95 & 29.11 & 0.93 & 3.24 & 1497.48 & 41.19 & 0.16 \\
\hline 3.06 & 129.47 & 29.27 & 1.25 & 3.46 & 1493.06 & 41.15 & 0.12 \\
\hline
\end{tabular}

Table 1. Global performance at low and high bit rates

Fig. 4 illustrates the improvement of subjective ROI quality using the proposed ROI- and tile-based controller. The example shows 
less block artifacts in the faces (a) than the reference (b). The facial expression is clearer and we can see better details.

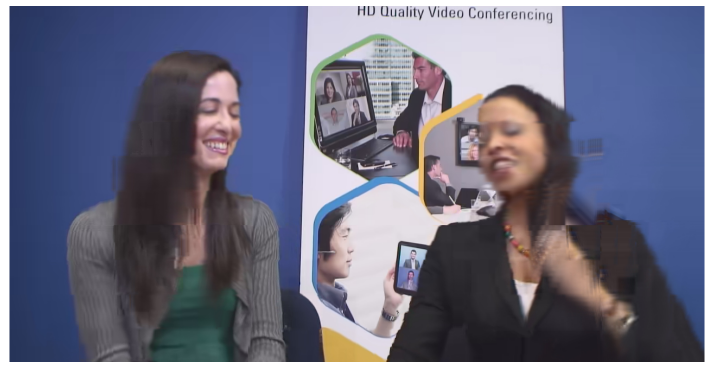

(a) Reference RC

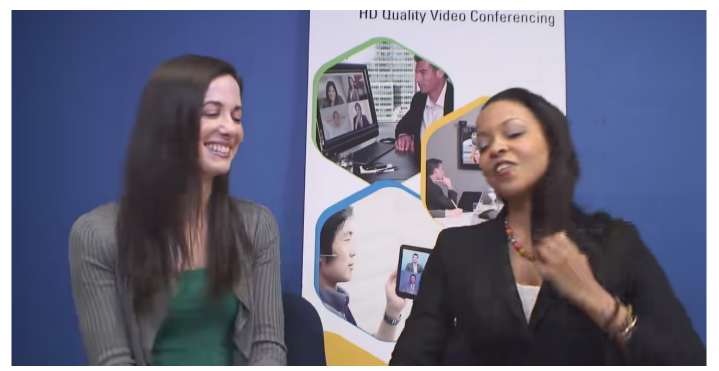

(b) ROI- and tile-based RC

Fig. 4. Comparison of subjective quality of "KristenAndSara" sequence encoded at $256 \mathrm{kbps}$ (Frame 593)

\subsection{ROI quality after decoding corrupted streams}

\begin{tabular}{|c||c|c|c||c|c|c|}
\hline \multicolumn{1}{|c||}{ Bit rates } & \multicolumn{3}{c||}{$128 \mathrm{kbps}$} & \multicolumn{3}{c|}{$1.5 \mathrm{Mbps}$} \\
\hline \hline Tested streams & 1 & 2 & 3 & 1 & 2 & 3 \\
\hline \hline "Johnny" & 29.50 & 30.26 & 31.44 & 34.10 & 39.24 & 39.32 \\
\hline "Kristen & 28.08 & 28.82 & 28.82 & 35.75 & 39.16 & 39.30 \\
AndSara" & & & & & & \\
\hline "FourPeople" & 25.16 & 23.72 & 24.90 & 31.82 & 37.28 & 37.37 \\
\hline
\end{tabular}

Table 2. PSNR ROI $(\mathrm{dB})$ of the three evaluated streams

Table 2 shows the PSNR (in $\mathrm{dB}$ ) of the ROI after decoding three corrupted streams per sequence. The first one (Stream 1) is coded using the reference HEVC controller, tiling is added in the second configuration (Stream 2) and the last stream (Stream 3) has been coded using the proposed algorithm (ROI- and tile-based RC algorithm). All streams have been transmitted through the same channel modeled as a Gilbert-Elliott channel (Table 3). The same packet loss pattern was then used for all evaluated video streams. Tests have been performed at both low and high bit rates.

\begin{tabular}{|c|c|c|c|}
\hline $1-p_{(G \rightarrow B)}$ & $1-p_{(B \rightarrow G)}$ & $e_{G}$ & $e_{B}$ \\
\hline 0.995 & 0.96 & $10^{-4}$ & $10^{-3}$ \\
\hline
\end{tabular}

Table 3. Gilbert-Elliot model parameters

For all tested sequences, we observe an improvement in ROI quality when using ROI- and tile-based RC (Stream 3) and comparing to the reference controller (Stream 1). When we loose a packet from the non-ROI, the ROI is not affected in our scheme and we have an average PSNR gain that can reach $5.5 \mathrm{~dB}$ ("FourPeople" at $1.5 \mathrm{Mbps}$ ).
In the worst case ("FourPeople" at $128 \mathrm{kbps}$ ), we loose a packet from the ROI, the ROI quality is almost the same using both reference and proposed schemes.

We notice from Table 1, that introducing a bit allocation factor $K$ between regions helps improve budget partitioning between tiled regions. Depending on the encoded sequence and the tiling, ROI- and tile-based RC (Stream 3) gives equivalent or improved quality of ROI comparing to tile-based reference controller (Stream 2).

If we check ROI quality frame by frame, we notice that when a packet is lost in the non-ROI, all ROIs of depending frames are affected in the reference scheme. On the contrary, our proposed scheme protects the ROI from non-ROI packet loss and corresponding tiles are not corrupted. This is visible in Fig.5, in particular we notice the error propagation affecting ROIs in the reference scheme.

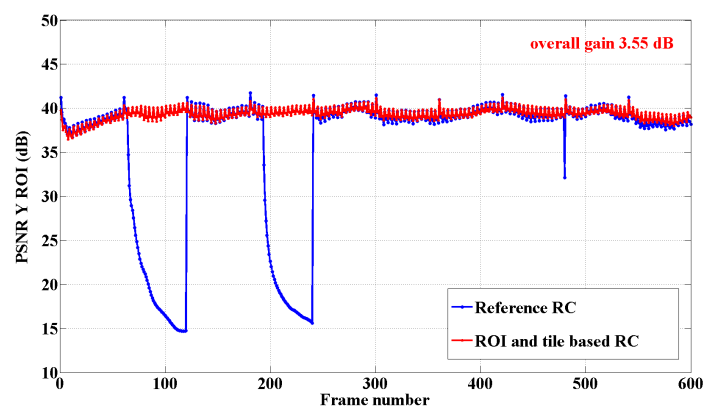

Fig. 5. PSNR ROI of "KristenAndSara" coded at 1.5 Mbps

Examples of the decoded class-E sequences available via the following link: http://cagnazzo.wp.mines-telecom.fr/en/?p=1092/ show the superiority of the proposed scheme with respect to the reference.

Finally, Table 4 shows that for different loss patterns the quality of the full sequence and the ROIs is much better using our method, even if some packets of the ROI are lost.

\begin{tabular}{|c||c|c||c|c|}
\hline \multirow{2}{*}{$\begin{array}{c}\text { Number of } \\
\text { lost packets }\end{array}$} & \multicolumn{2}{c||}{ Reference } & \multicolumn{2}{c|}{ Proposed } \\
\cline { 2 - 5 } & PSNR $(\mathrm{dB})$ & PSNR ROI $(\mathrm{dB})$ & PSNR $(\mathrm{dB})$ & PSNR ROI $(\mathrm{dB})$ \\
\hline 4 & 37.34 & 35.75 & 39.21 & 39.30 \\
\hline 5 & 36.89 & 35.44 & 39.50 & 39.03 \\
\hline 7 & 35.91 & 33.81 & 37.86 & 37.13 \\
\hline
\end{tabular}

Table 4. Comparison of "KristenAndSara" decoding quality at 1.5 Mbps using different loss patterns

\section{CONCLUSION}

In this paper, an ROI- and tile-based rate control algorithm for HEVC has been proposed. Implementation has been done in HM.13 and the controller performance has been evaluated at video coding layer and network layer. The scheme achieves better visual quality in ROIs thanks to independent rate allocation between regions, encoding and transmission of regions. At VCL layer the QP of LCUs of the same region are independently computed from the rest of the frame, the regions are coded in separate tiles and then transmitted in different NAL units. Consequently, at NAL layer, transmission errors do not affect both regions, they are limited to the affected tile and depending tiles at the same spatial position. As a conclusion, this scheme allows a better representation of the ROI while respecting the global rate constraint. 


\section{REFERENCES}

[1] Z. Wu, S. Xie, K. Zhang, and R. Wu, "Rate Control in Video Coding," in Recent Advances on Video Coding, J. Del Ser Lorente, Ed. InTech, 2011, pp. 79-117.

[2] L. Yang, L. Zhang, S. Ma, and D. Zhao, "A ROI quality adjustable rate control scheme for low bitrate video coding," in Proceed. of Pict. Cod. Symp. IEEE, May 2009, pp. 1-4.

[3] J. Chiang, C. Hsieh, G. Chang, F.-D. Jou, and W.-N. Lie, "Region-of-interest based rate control scheme with flexible quality on demand," in Proceed. of IEEE Intern. Conf. on Multim. and Expo, 2010, pp. 238-242.

[4] C.-Y. Wu and P.-C. Su, "A region-of-interest rate-control scheme for encoding traffic surveillance videos," in International Conference on Intelligent Information Hiding and Multimedia Signal Processing, 2009, pp. 194-197.

[5] H. Hu, B. Li, W. Lin, W. Li, and M.-T. Sun, "Region-based rate control for H.264/AVC for low bit-rate applications," IEEE Trans. Circuits Syst. Video Technol., vol. 22, no. 11, pp. 1564 1576, Nov. 2012.

[6] G. J. Sullivan, J.-R. Ohm, W.-J. Han, and T. Wiegand, "Overview of the high efficiency video coding (HEVC) standard," IEEE Trans. Circuits Syst. Video Technol., vol. 22, no. 12, pp. 1649-1668, 2012.

[7] K. Misra, A. Segall, M. Horowitz, S. Xu, A. Fuldseth, and M. Zhou, "An overview of tiles in hevc," Selected Topics in Signal Processing, IEEE Journal of, vol. 7, no. 6, pp. 969-977, Dec 2013.

[8] M. Meddeb, M. Cagnazzo, and B. Pesquet-Popescu, "Region-ofInterest Based Rate Control Scheme for High Efficiency Video Coding," in Proceed. of IEEE Intern. Conf. Acoust., Speech and Sign. Proc., Florence, Italy, 2014.

[9] _ _ "Region-of-Interest Based Rate Control Scheme for High Efficiency Video Coding," APSIPA Transactions on Signal and Information Processing, vol. 3, 2014.

[10] B. Li, H. Li, L. Li, and J. Zhang, "Rate control by R-lambda model for HEVC," in JCT-VC K0103, 11th meeting of Joint Collaborative Team on Video Coding of ITU-T SG 16 WP 3 and ISO/IEC JTC 1/SC 29/WG 11, Shanghai, CN, 10-19 Oct. 2012.

[11] B. Li, H. Li, and L. Li, "Adaptive bit allocation for R-lambda model rate control in HM," in JCT-VC M0036, 13th meeting of Joint Collaborative Team on Video Coding of ITU-T SG 16 WP 3 and ISO/IEC JTC 1/SC 29/WG 11, Incheon, KR, 18-26 Apr. 2013.

[12] HEVC test model 13 (HM.13). [Online]. Available: https://hevc.hhi.fraunhofer.de/svn/svn_HEVCSoftware/tags/HM$13.0 /$

[13] G. Hasslinger and O. Hohlfeld, "The Gilbert-Elliott model for packet loss in real time services on the Internet," Measuring, Modelling and Evaluation of Computer and Communication Systems (MMB), 2008 14th GI/ITG Conference, 2008.

[14] J. Ebert and A. Willig, "A Gilbert-Elliot Bit Error Model and the Efficient Use in Packet Level Simulation," tkn.tu-berlin.de, 1999.

[15] H. Choi, J. Nam, J. Yoo, and D. Sim, "Rate control based on unified RQ model for HEVC," in JCT-VC H0213, 8th meeting of Joint Collaborative Team on Video Coding of ITU-T SG 16 WP 3 and ISO/IEC JTC 1/SC 29/WG 11, San José, CA, USA, 2012.
[16] — - "Improvement of the rate control based on pixel-based URQ model for HEVC," in JCT-VC I0094, 9th meeting of Joint Collaborative Team on Video Coding of ITU-T SG 16 WP 3 and ISO/IEC JTC 1/SC 29/WG 11, Geneva, CH, 2012.

[17] M. Karczewicz and X. Wang, "Intra Frame Rate Control Based SATD," in JCT-VC M0257, 13th meeting of Joint Collaborative Team on Video Coding of ITU-T SG 16 WP 3 and ISO/IEC JTC 1/SC 29/WG 11, Incheon, KR, 2013.

[18] B. Bross, W.-J. Han, J.-R. Ohm, G. J. Sullivan, Y.-K. Wang, and T. Wiegand, "High Efficiency Video Coding (HEVC) text specification draft 10," in JCT-VC L1003, 12th meeting of Joint Collaborative Team on Video Coding of ITU-T SG 16 WP 3 and ISO/IEC JTC 1/SC 29/WG 11, Geneva, CH, 2013.

[19] M. B. Vivienne Sze, Gary J. Sullivan, High Efficiency Video Coding (HEVC) - Algorithms and architectures. Springer, 2014.

[20] Y. Ye, Y. He, and Y. He, "SEI message: independently decodable regions based on tiles," in JCT-VC L0049, 12th meeting of Joint Collaborative Team on Video Coding of ITU-T SG 16 WP 3 and ISO/IEC JTC 1/SC 29/WG 11, Geneva, CH, 2013.

[21] — "ROI tile sections," in JCT-VC K0103, 11th meeting of Joint Collaborative Team on Video Coding of ITU-T SG 16 WP 3 and ISO/IEC JTC 1/SC 29/WG 11, Shanghai, CN, 2012.

[22] R. Gregory-Clarke, L. D’Acunto, R. van Brandenburg, E. Thomas, G. Thomas, and O. Niamut, "Immersive Live event Experiences - Interactive UHDTV on Mobile Devices," The best of IET and IBC, vol. 6, pp. 38-43, 2014.

[23] S. Brangoulo, N. Tizon, B. Pesquet-Popescu, and B. Lehembre, "Video Transmission over UMTS Networks Using UDP/IP," in Proceed. of Europ. Sign. Proc. Conf., Florence, Italy, 2006, pp. 3-7.

[24] F. Bossen, "Common test conditions and software reference configurations," in JCT-VC L1100, 12th meeting of Joint Collaborative Team on Video Coding of ITU-T SG 16 WP 3 and ISO/IEC JTC 1/SC 29/WG 11, Geneva, CH, 2013. 